\title{
NEW ECLIPSING PHENOMENA OF THE CENTRAL STAR IN
}

NGC 2346

R. COSTERo, M. PEÑA, W.J. SCHUSTER, M. TAPIA, J. ECHEVARRIA and J. FIERRO

Instituto de Astronomía, UNAM, Mexico

The central star of NGC 2346 is a well known binary with an A-type primary and a hot companion (Méndez and Niemela 1981). The star went through a series of periodic light variations which ceased in 1986 and were interpreted as an eclipse of a dust cloud passing in front of the binary system (e.g. Méndez et al. 1982, Costero et al. 1986). Recently, light variations reappeared with shallower minima compared to the previous eclipse (e.g. Kohoutek et al. 1992).

We have made photoelectric and CCD photometry of this star (V651 Mon) at the Observatorio Astronómico Nacional in San Pedro Mártir, Mexico, during April/May 1992. An eclipse of amplitude $\sim 0.25$ in the observed V magnitude was measured, similar to previous reports, but now it seems to be deeper and broader. We interpret this variations in terms of another fragmented dust cloud passing in front of the central star.

\section{References}

Costero R., Tapia M., Méndez R.H., Echevarria J., Roth M., Quintero A., Barral J.F. 1986, Rev. Mexicana Astron. Astrof. 13, 149.

Kohoutek L., Mantegazza L., Hainaut, O. 1992, Inf. Bull. Var. Stars No. 3694.

Méndez R.H., Niemela V.S. 1981, ApJ 250, 240.

Méndez R.H., Gathier R., Niemela V.S. 1982, A\&A 116, L5. 